\title{
COMMUNICATION IN SOCIAL DISTANCING: THE ROLE OF TECHNOLOGY IN COMMUNICATION TO OVERCOME SOCIAL ISOLATION
}

\author{
Cherlydea Gladys ${ }^{1 *}$, Yohanes Muljono ${ }^{2}$, Bambang Irwanto ${ }^{3}$ \\ ${ }^{*}$ Communication Communication, Institue of London School of Public Relations, Jakarta, Indonesia \\ ${ }^{23}$ Communication Communication, Institue of London School of Public Relations, Jakarta, Indonesia \\ *Email: cherlydeagladyss@gmail.com
}

\begin{abstract}
Social distancing aims to reduce interactions between people in the wider community, where individuals may have been infected or are carriers but have not been identified so as not to be isolated. The impact of social distancing is social isolation due to reduced social interaction. Related to social distancing issues as the impact of infectious disease outbreak has the potential to cause social isolation, it is known that the development of information and communication technology can overcome social and spatial barriers of social interaction by enabling easier communication processes. The results revealed that the development of technology has an important role as a medium of communication in social distancing problems as the impact of infectious disease outbreak has the potential to cause social isolation.
\end{abstract}

Keywords: communication, social distancing, social isolation, technology

\section{INTRODUCTION}

The term social distancing has emerged as the main line of defense in the human struggle against the new corona virus. The background of social distancing is considered convincing and clear, because people infected with coronavirus can transmit it up to five days before symptoms appear, or even transmit without symptoms. Thus, it was concluded that reducing social interactions between even healthy people could dramatically slow the spread of the virus. Critical cases can thus remain at a number low enough to be treated effectively in intensive care units. Prior to novel coronavirus (2019-nCoV), in 2003 an influenza pandemic was also occurring, the severe acute respiratory syndrome (SARS) which encouraged policy recommendations that encourage social distance to be considered effective against influenza pandemics.

Social distancing is considered to be very useful in settings where community transmission is believed to have occurred, but it is still unclear where the relationship between cases, and where restrictions are placed only on people who are known to be exposed are considered insufficient to prevent further transmission. Examples for social alignment include the closure of schools or office buildings and public market suspensions, and the cancellation of meetings. Social distancing has become a very important aspect of human behavior for epidemiology because of its universal nature, where everyone can reduce the level of contact with others by changing their behavior, and reduce human contact which can help reduce the transmission of many diseases.

Everyone reacts differently to stressful situations such as epidemics of infectious diseases that require the imposition of social distance, where people will feel anxiety, worry, or fear. Anxiety, loneliness, to depression and stress will usually arise when someone does social isolation in social distancing. Social isolation is considered as a result of reduced social interaction, especially with family, friends, and community networks. Because social isolation usually occurs in the elderly, things that cause it to occur by 
retirement, physical changes, loss of spouse or friends resulting in shrinking network size, and living alone or in institutions.

In general when the number of relationships in a private network increases, the intensity of loneliness as one of the effects of social isolation will also decrease. Those who have a small personal network and those who are on the margins of social networking have the greatest risk for loneliness, lonely people tend to accept less friendship from others. Related to social distancing issues as the impact of infectious disease outbreak has the potential to cause social isolation, it is known that the development of information and communication technology can overcome social and spatial barriers of social interaction by enabling easy and affordable communication through various forms of activities such as text, audio or visual between people who are isolated or experience social distancing with limited mobilization with others anytime and anywhere. Based on this background, this paper will discuss technological developments in communication in the face of social isolation.

\section{METHOD}

This research is a descriptive qualitative social study. Qualitative descriptive method is a method used to describe or analyze a research result but is not used to make broader conclusions. The qualitative research method itself is a research method used to examine the condition of natural objects by conducting literature studies and observations. The data collected is then analyzed by descriptive method, a presentation of the analysis through interpretation accompanied by a rational interpretation of the factors in the field.

\subsection{Data Collection}

Data collection techniques used in this research are observation and study of literature. Observation technique is a data collection technique used to collect research data through observation and sensing the behavior of human life, work processes, and natural phenomena and others to be observed and known what happens in the life of the community. The method of observation is used to collect data about the situations and conditions of everyday life, that is, how people communicate in this social distancing situation. Observation technique is used in data collection, because this technique can reveal and describe the communication carried out by the community during social distancing.

The literature study technique is used by tracing the literature that contains theories from published or unpublished scientific works in the form of hard copies or soft copies in e-books, papers, and online journals. This technique is used as a primary data collection technique in testing the esearch results obtained about the communication patterns used by the community during social distancing. Literature studies are used as a technique in research to obtain data because the data obtained in the form of theories that support research activities, and the data obtained later are used to verify the quality of theories found from the results of research.

\section{RESULT AND DISCUSSION}

\subsection{Infectious disease outbreak and social distancing}

Infectious diseases will usually be controlled by minimizing contact between infectious and vulnerable individuals. Personal actions aimed at reducing contact that have the potential to be transmitted are called social distancing. It has been suggested that policies that encourage social distance might be effective against influenza pandemics. These forms of influenza pandemic include the severe acute respiratory syndrome (SARS), which spread throughout the world in 2003, is one of the new infectious diseases that emerged in the 21 st century and the novel 
coronavirus (2019-nCoV) which appeared in Wuhan, China in December 2019 which has a very rapid spread in Hubei province and now has reached all provinces in China and has also spread to more than 20 countries. Previously, SARS was considered scary at the time, perhaps even more frightening compared to 2019-n CoV because it progressed far more frequently to severe illness and death. But the world is able to completely stop human-to-human transmission, stop epidemics.

Public health actions are crucial in controlling the SARS epidemic in 2003. And in 2020 the world is facing a situation of epidemics with viruses that do not have special vaccines. Thus a way is needed to rely on public health measures. The main purpose of public health action is to prevent the spread of disease from human to human by separating people to stop transmission, which can be done with isolation, quarantine, social distance, community detention. All of these activities are on a large scale that has never happened before. Isolation is the separation of the sick from people who are not infected. Quarantine is a restriction of movement, often with fever control, contact when it is unclear whether they have been infected but have not shown symptoms or have not been infected. Community detention includes measures that range from increasing social distance to quarantine throughout the community.

Social distancing aims to reduce interactions between people in the wider community, where individuals may have been infected or are carriers but have not been identified so as not to be isolated. Because diseases transmitted by respiratory droplets require a certain closeness to people, social distance between people will reduce transmission. Social distancing is considered to be very useful in settings where community transmission is believed to have occurred, but it is still unclear where the relationship between cases occurs, and where restrictions are placed only on people known to be exposed are considered not enough to prevent further transmission. Examples for social alignment include the closure of schools or office buildings and public market suspensions, and the cancellation of meetings.

\subsection{Social distancing and social isolation during pandemic}

Social distancing has become a very important aspect of human behavior for epidemiology because of its universal nature, where everyone can reduce the level of contact with others by changing their behavior, and reducing human contact which can help reduce the transmission of many diseases. The theoretical work on social distance has been stimulated by agent-based influenza simulation studies which show that small changes in behavior can have a large effect on transmission patterns during epidemics. Social distancing refers to the adoption of behavior by individuals in a community that reduces the risk of the individual being infected by limiting their contact with other individuals or reducing the risk of transmission during each contact. Usually, social distancing can have an impact in terms of freedom, social capital, time, comfort, and money, so people will only adopt these steps when there is a special incentive to do so. In addition to personal consequences, the aggregate effect of social distance forms economic externalities, reducing overall disease transmission.

Everyone reacts differently to stressful situations such as epidemics of communicable diseases that require social distance to be imposed, where people will feel anxiety, worry, or fear related to the health status of themselves or others who may have been exposed to the disease , time taken from work and potential loss of income and job security, challenges to secure the things needed, uncertainty or frustration about how long the situation will last, loneliness related to feeling disconnected from the world and from 
loved ones, boredom and frustration because it may not be able to work or engage in daily routine activities, uncertainty about the situation, symptoms of depression, to symptoms of stress disorder. Anxiety, loneliness, to depression and stress will usually arise when someone does social isolation in social distancing. Some researchers reveal the impact of social distance, where there are social potential and psychological impacts. It was stated that over a long period of time, social isolation was considered to be able to increase the risk of various health problems, including heart disease, depression, dementia, and even death. This is because social contact can protect the negative effects of stress. Holt-Lunstad mentions that people of all ages are considered vulnerable to the ill effects of social isolation and loneliness. The National Academy of Sciences emphasizes that older people may be more vulnerable to the effects of social isolation.

$$
\text { Social isolation is a }
$$

multidimensional concept that does not have a clear and consistent definition. There is an opinion that sees it directly as being equivalent to loneliness and uses both terms by changing. While other opinions consider the two concepts related but different. Social isolation has been defined as the absence of contact with people who provide social support. Others define it as a two-dimensional concept that contains the absence of objective contact or interaction with contact and subjective feelings of friendship or limited or lost social support resulting from having limited contact or interaction. Social isolation is considered as a result of reduced social interaction, especially with family, friends, and community networks. Because social isolation usually occurs in the elderly, things that cause it to occur by retirement, physical changes, loss of spouse or friends resulting in shrinking network size, and living alone or in institutions. Based on the criteria for the causes of social isolation, it is related to social distancing as a result of infectious disease outbreaks such as SARS and 2019$\mathrm{nCoV}$, so people who are in social distancing coverage have the potential to experience social isolation. This is because in social distancing, they tend to reduce social interaction, especially with family, friends, and community networks they have, or even live alone or more often alone because of isolation that forces them to stay at home. Extreme situations that cause them to experience social isolation are the loss of a partner or friend resulting in shrinking network size. Of all these criteria, it can be concluded that social distancing as a result of infectious disease outbreak has the potential to cause social isolation due to the lack of contact or social interaction carried out by individuals.

Social isolation is an objective and measurable reflection of the reduced size of social networks and the lack of social contact. This is a special problem when there are economic resources, decreased mobility, and all kinds of conditions that cause restrictions on social contact. Socially isolated individuals are at increased risk for developing a number of diseases. Social isolation concerns the objective characteristics of a situation and refers to a small network of relatives and non-relatives. There is a continuum from social isolation at one end to social participation on the other. People with absences or a small number of meaningful ties are socially isolated. Research shows that people who are socially isolated have a greater risk of becoming lonely, because social networking of personal relationships has a very important role to feel socially embedded and to reduce loneliness.

In general when the number of relationships in a private network increases, the intensity of loneliness as one of the effects of social isolation will also decrease. Those who have a small personal network and those who are on the margins of social networking have the greatest risk for loneliness, lonely people tend to accept 
less friendship from others. Loneliness is only one of the possible outcomes of evaluating a situation marked by a small number of relationships. People who are socially isolated will not necessarily experience loneliness, and lonely people will not necessarily be socially isolated in an objective sense. Individuals will end up on a subjective continuum depending on expectations or the standard of the relationship. Individuals with a small amount of social contact may feel lonely. The size of the private network is considered important, but many other aspects of the network are worth mentioning too, such as the composition and function of the network. Individuals with a network of strong and weak ties are less susceptible to loneliness than individuals with strong bonds. And individuals with networks that include family ties will be more vulnerable to loneliness compared to individuals with heterogeneous networks, which include both relatives and non-relatives.

Social alienation, such as loneliness is a concept that is understood or often described as an objective state associated with the subjective concept of loneliness and some claim that loneliness itself becomes a subjective part of social isolation. At the most basic level, social isolation has been defined as a lack of meaningful and ongoing communication, or as having minimal contact with family or the wider community. Various approaches to measuring isolation involve recording the level of social contact, counting social participation and measuring social networking. The nature of a person's social network has been identified as a key level of social isolation that they experience. Networks are described as identified social relationships that surround a person, their characteristics, and individual perceptions about them. Social networks can be identified by size, that is the number of people in the network, the density of the extent to which network members are interrelated and accessibility and reciprocity.

\subsection{The role of technology in communication to overcome social isolation}

Humans communicate with the aim of survival and development. Communication has an important function to share resources and information and to create alliances. Historically, the people who are most important to communicate are the closest people, ie potential allies or spouses. Indeed, spatial closeness is a reliable predictor of whether two people will encourage social relations. Initially people can only communicate in real time with people in close spatial distance. But along with the development of technology, telephones and cellular phones have emerged which are rapidly improving realtime long distance communication. As of July 2015, 92\% of American adults have cellphones, by bridging physical distance, mediated communication can reduce social distance. Mediated communication can even encourage greater intimacy compared to face-to-face communication. After making a phone call, people feel socially closer to their conversation partners than people at their actual physical location.

Related to social distancing issues as the impact of infectious disease outbreak has the potential to cause social isolation, it is known that the development of information and communication technology can overcome social and spatial barriers of social interaction by enabling easy and affordable communication through various forms of activities such as text, audio or visual between people who are isolated or experience social distancing with limited mobilization with others anytime and anywhere. The important role of technological development for social connectivity starts from the beginning of this millennium, as explained in Mynatt et al. and Hindus et al. on digital family portraits and social communication devices for the home, where technological 
development is closest to the design of communication equipment for intimate social networking. The last few decades technological developments emphasize the use of technology with the aim of supporting domestic communication very different from traditional media spaces designed for the workplace. Because the technological development is more focused on connections its own interests, and not as a means towards instrumental goals such as project coordination or editing documents.

Tsai and Tsai revealed that the development of these technologies supports the communication that is done through videoconferencing interactions with families. And these activities are proven to reduce the symptoms of depression and loneliness as a result of social isolation in which individuals tend to live alone and low social interaction with other individuals. This form of renewable communication will have a positive impact on individuals where they will avoid loneliness, they are cognitively healthy which is a form of improved physical well-being and health, social connectedness, and the ability to stay at home. There are patterns of communication and interpersonal involvement that are independent of technology. Technology is perceived as a tool for managing and adapting to changing circumstances and the ability to carry out social interactions.

Regarding technological developments, smartphone usage and penetration continues to increase, there is an important role in smartphone use in social isolation by strengthening social capital. According to Coleman, social capital depends on human relationships that can function as resources for future benefits. Social capital is conceptualized in various ways. Putnam and Fukuyama revealed three main components of social capital at the community level focused on trust, networking, and reciprocity. Burt and Portes revealed that there is a significant relationship between social networks and social capital. In contrast to this approach, Lin emphasizes the importance of individual interaction to build social capital.

Related to a significant relationship between the use of smartphone applications and social capital, there is the potential effects of social capital on an individual's personal life. Thus, there is a potential role for social capital to reduce social isolation. This is because social capital is determined by the personal interaction of individuals and social relationships with others who have influence. This implies the potential function of social capital to strengthen individual social connections. Related to this issue, Ledbetter et al. revealed the positive effects of using online social media such as Facebook on relational closeness, and social capital can increase one's feeling of social support. Increased personal ties and close relationships are associated with a reduction in social isolation. According to Seeman, social isolation is one of the four dimensions of social alienation. Isolation relates to people who set low prize values for goals or beliefs that are usually highly valued in certain societies. As such, individuals who are socially isolated rarely share the values that are usually accepted by others in their community. Therefore, as these isolated individuals get more opportunities to communicate with other influential people and strengthen ties and bridge social capital, they will be more likely to share the general values of their society.

Electronic social networks, such as Facebook, Twitter, Instagram, and others, are the most popular websites or applications in recent years. Facebook has become the largest social network in the world, with more than half a billion active users and $130 \%$ growth in the 12 months before 2010. Facebook is a very active network with more than 250 million users entering the network at least once a day. The average user on Facebook has 130 
members and they spend 47 minutes on the network. More than 35 million users update their status at least once a day and more than 30 billion content items are uploaded every month. There are studies that find that lonely people have a tendency to use the internet and email intensively. This is because the media is considered to be able to provide them with emotional support from others. The online social behavior of lonely people is usually consistent and they will get satisfaction with their relationships with online friends. These people have a tendency to use the internet to overcome negative moods, even though they reveal that internet use can also disrupt daily functions and spend the time they want to invest.

As a result of technological developments, communication applications on smartphones have a positive influence in bridging social capital. In addition, Park et al. revealed that smartphone usage significantly has a positive impact on social capital by being mediated by the use of online social media applications. Bonding and bridging social capital can significantly reduce social isolation. These results re-emphasize the importance of social capital, especially the bonds of social capital, to improve the personal lives of individuals. In addition, on the basis of considering the positive effects of using smartphone applications on social capital, the results of the study emphasize the use of smartphone applications, especially communication applications and online social media to improve people's lives, especially among younger populations. The findings of this study contribute to broadening understanding of the realistic role of smartphone use as a form of technological development in overcoming social isolation problems. Thus the development of technology has an important role as a medium of communication in social distancing issues as the impact of infectious disease outbreak has the potential to cause social isolation.

\section{CONCLUSION}

Infectious diseases will usually be controlled by minimizing contact between infectious and vulnerable individuals. Personal actions aimed at reducing contact that have the potential to be transmitted are called social distancing. Social distancing aims to reduce interactions between people in the wider community, where individuals may have been infected or are carriers but have not been identified so as not to be isolated. Everyone reacts differently to stressful situations such as epidemics of infectious diseases that require the imposition of social distance, where people will feel anxiety, worry, or fear. Anxiety, loneliness, to depression and stress will usually arise when someone does social isolation in social distancing. Social isolation is considered as a result of reduced social interaction, especially with family, friends, and community networks.

Related to social distancing issues as the impact of infectious disease outbreak has the potential to cause social isolation, it is known that the development of information and communication technology can overcome social and spatial barriers of social interaction by enabling easier communication processes through various forms of activities such as text, audio or visual between people who are isolated or experience social distancing with limited mobilization with others anytime and anywhere. Regarding technological developments, smartphone usage and penetration continues to increase, there is an important role for smartphone use in social isolation by strengthening social capital. Communication applications on smartphones and online social media have a positive influence in bridging social capital. Bonding and bridging social capital can significantly reduce social isolation. Thus the development of technology has an important role as a medium of communication in social distancing issues as the impact of 
infectious disease outbreak has the potential to cause social isolation.

\section{REFERENCES}

Baecker, R., Sellen, K., Crosskey, S., Boscart, V., \& Neves, B. B. (2014). Technology to Reduce Social Isolation and Loneliness. Proceedings of the 16th International ACM SIGACCESS Conference on Computers \& Accessibility - ASSETS '14.

Caley, P., Philp, D. J., \& McCracken, K. (2007). Quantifying social distancing arising from pandemic influenza. $J . R$. Soc. Interface.5631-639.

Chen, Y.-R. R., \& Schulz, P. J. (2016). The Effect of Information Communication Technology Interventions on Reducing Social Isolation in the Elderly: A Systematic Review. J Med Internet Res 2016 | vol. $18 \mid$ iss. 1 | e18.

Cho, J. (2015). Roles of Smartphone App Use in Improving Social Capital and Reducing Social Isolation. Cyberpsychology, Behavior, And Social Networking Volume 18, Number 6, 2015.

Gierveld, J. d., Tilburg, T. G., \& Dykstra, P. A. (2016). Loneliness and Social Isolation. In A. Vangelisti, \& D. Perlman, The Cambridge Handbook of Personal Relationships, second edition. Cambridge: Cambridge University Press.

Gil-Or, O. (2011). Social networks and social isolation - A reason and its solution in one. Journal of Management Volume 2 Issue 1.

Long, N. J. (2020). From social distancing to social containment: reimagining sociality for the coronavirus pandemic. Medicine Anthropology Theory.

Miller, G. (2020). Social distancing prevents infections, but it can have unintended consequences. Retrieved March 28, 2020, from Science Mag: https://www.sciencemag.org/news/2020 /03/we-are-social-species-how-willsocial-distancing-affect-us\#

Reluga, T. C. (2010). Game Theory of Social Distancing in Response to an Epidemic. PLoS Comput Biol. 2010 May; 6(5).

Steptoe, A., Shankar, A., Demakakos, P., \& Wardle, J. (2013). Social isolation, loneliness, and all-cause mortality in older men and women. PNAS April 9, 2013110 (15) 5797-5801.

Substance Abuse and Mental Health Services Administration. (2014). Tips For Social Distancing, Quarantine, And Isolation During An Infectious Disease Outbreak. HHS Publication No. SMA14-4894 (2014).

Victor, C., Scambler, S., Bond, J., \& Bowling, A. (2000). Being alone in later life: loneliness, social isolation and living alone. Reviews in Clinical Gerontology 2000 10; 407-417.

Won, A. S., Shriram, K., \& Tamir, D. I. (2017). Social Distance Increases Perceived Physical Distance. Social Psychological and Personality Science 1-9.

Wilder-Smith, A., \& Freedman, D. O. (2020). Isolation, quarantine, social distancing and community containment: pivotal role for old-style public health measures in the novel coronavirus (2019-nCoV) outbreak. Journal of travel medicine, 27(2). 